\title{
OPEN A New Tool for In Vivo Study of Astrocyte Connexin 43 in Brain
}

\author{
Marine Droguerre ${ }^{1,7}$, Tomokazu Tsurugizawa ${ }^{2,7}$, Adeline Duchêne ${ }^{1}$, Benjamin Portal ${ }^{3}{ }^{3}$, \\ Bruno P. Guiard ${ }^{3}$, Nicole Déglon $\mathbb{1}^{4,5}$, Nathalie Rouach ${ }^{6}$, Michel Hamon ${ }^{1}$, Franck Mouthon ${ }^{1}$, \\ Luisa Ciobanu ${ }^{2 *} \&$ Mathieu Charvériat $\mathbb{1 0}^{1 *}$
}

Astrocytes are glial cells organized in dynamic and structured networks in the brain. These plastic networks, involving key proteins such as connexin 43 (Cx43), are engaged in fine neuronal tuning and have recently been considered as emerging therapeutic targets in central nervous system disorders. We developed and validated a new application of the manganese-enhanced magnetic resonance imaging (MEMRI) technique allowing in vivo investigations of astrocyte-neuron interactions through quantification of brain Cx43 functional activity. The proof of concept has been achieved by quantification of MEMRI signals in brain after either local astrocyte-specific Cx43 knockdown with shRNA or systemic administration of Cx43 blockers. Unilateral hippocampal Cx43 genetical silencing was associated with an ipsilateral local increase of MEMRI signal. Furthermore, Cx43 blockers also enhanced MEMRI signal responses in hippocampus. Altogether, these data reveal the MEMRI technique as a tool for quantitative imaging of in vivo $\mathrm{Cx} 43$-dependent function in astrocytes under physiological and pathological conditions.

Data accumulated over the last decades mainly point out a role for glial cells in providing neurons with metabolic, structural and trophic support ${ }^{1}$. However, recent data have also shown that astrocytes are key non-neuronal cells involved in active signaling contributing to brain functions, as they notably regulate sleep-wake cycle, $\operatorname{cognition}^{2}$ and behavior ${ }^{3}$. At the cellular level, neuronal activity is tuned by astrocytes, in particular at tripartite synapses, that associate pre- and postsynaptic structures with an astroglial element ${ }^{4}$. Furthermore, astrocytes are organized in tightly regulated plastic networks ${ }^{5}$, through the transmembrane proteins connexins $(\mathrm{Cx})$, especially connexin 43 (Cx43), which form hemichannels and gap junction channels ${ }^{6}$. A large body of evidence already shows that these networks are involved in neuronal modulation and play important roles in brain functions $\mathrm{s}^{3,7-9}$.

To date, only few imaging markers of astrocytes such as positron emission tomography (PET) probes ${ }^{10}$, passive fluorescent dyes ${ }^{11}$ and genetically encoded calcium ions $\left(\mathrm{Ca}^{2+}\right)$ indicators ${ }^{12}$, have been developed and successfully used. However, the in vivo usefulness of these probes is limited due to the requirement of their local intracerebral application ${ }^{13}$. In vivo brain imaging of the interactions between astrocytes and neurons is hence still challenging. We herein report data showing that manganese-enhanced magnetic resonance imaging (MEMRI) can capture those interactions. Indeed, previous findings using MEMRI suggest that: (i) manganese enters both neurons and astrocytes in brain ${ }^{14}$, (ii) astrocytes concentrate $80 \%$ of cerebral manganese ions $\left(\mathrm{Mn}^{2+}\right)^{15}$, and (iii) $\mathrm{Mn}^{2+}$ shares chemical similarities with $\mathrm{Ca}^{2+}$, thus being able to enter neurons and astrocytes through $\mathrm{Ca}^{2+}$ channels and $\mathrm{Na}^{+} / \mathrm{Ca}^{2+}$ exchangers $^{14}$. Accordingly, glial activation, notably during neuro-inflammatory processes ${ }^{16}$, neuronal tract tracing, and other specifically neuronal features could be studied using MEMRI. In our study, we developed and validated a new application of the MEMRI technique allowing the direct in vivo investigation of astrocyte-neuron interactions through quantification of hippocampus $\mathrm{Cx} 43$ functional activity. To this goal, we used in vivo administration of recombinant lentivirus targeting $\mathrm{Cx} 43$ expression and systemic treatments with known connexin modulators in mice.

\footnotetext{
${ }^{1}$ Theranexus, 60 Avenue Rockefeller, 69008, Lyon, France. ${ }^{2}$ NeuroSpin, CEA, 91191, Gif-sur-Yvette, France. ${ }^{3}$ Centre de Recherches sur la Cognition Animale (CRCA), Centre de Biologie Intégrative (CBI), Université de Toulouse, CNRS, UPS, 31330, Toulouse, France. ${ }^{4}$ Laboratory of Neurotherapies and NeuroModulation, Neuroscience research Center (CRN), Lausanne University Hospital (CHUV) and University of Lausanne, 1011, Lausanne, Switzerland. ${ }^{5}$ Laboratory of Neurotherapies and NeuroModulation, Department of Clinical Neuroscience (DNC), Lausanne University Hospital (CHUV) and University of Lausanne, 1011, Lausanne, Switzerland. ' ${ }^{6}$ Laboratory of Neuroglial Interactions in Cerebral Physiopathology, Center for Interdisciplinary Research in Biology, Collège de France, CNRS UMR 7241, INSERM U1050, Labex Memolife, PSL Research University, Paris, 75005, France. ${ }^{7}$ These authors contributed equally: Marine Droguerre and Tomokazu Tsurugizawa. *email: luisa.ciobanu@cea.fr; mathieu.charveriat@theranexus.com
} 


\begin{tabular}{|l|l|l|l|l|}
\hline & \multicolumn{2}{|l|}{$\begin{array}{l}\text { Acquisition } \\
\text { time (min) }\end{array}$} & \multicolumn{2}{l|}{ Spatial resolution } \\
\cline { 2 - 5 } & $\mathbf{7 T}$ & $\mathbf{1 1 . 7 ~ T}$ & $\mathbf{7 T}$ & $\mathbf{1 1 . 7 ~ T}$ \\
\hline RARE & 80 & 47 & $0.1 \times 0.1 \times 0.1 \mathrm{~mm}^{3}$ & $0.1 \times 0.1 \times 0.1 \mathrm{~mm}^{3}$ \\
\hline FISP & 60 & 70 & $0.15 \times 0.15 \times 0.15 \mathrm{~mm}^{3}$ & $0.1 \times 0.1 \times 0.1 \mathrm{~mm}^{3}$ \\
\hline
\end{tabular}

Table 1. Data acquisition parameters for the two MRI devices (7 T and 11.7 T). RARE, 3D Rapid Acquisition with Relaxation Enhancement; FISP, Fast Imaging with Steady-state free Precession.

\section{Materials and Methods}

Animals. Experiments were performed on 42 wild-type C57BL/6 male mice. Mice were housed 4-5 per cage under standard conditions (12/12 h light-dark cycle, light on at $7 \mathrm{am}, 22 \pm 1{ }^{\circ} \mathrm{C}$ ambient temperature, $60 \%$ relative humidity). Animal surgery and experimentations conducted in this study were approved by (i) the French Minister (Ministère de l'Education Nationale, de l'Enseignement Supérieur de la Recherche; France) and (ii) the local ethics committee (Comité d'Ethique en Expérimentation Animale, Commissariat à l'Energie Atomique et aux Energies Alternatives, Direction des Sciences du Vivant; Fontenay-aux-Roses, France) under reference: APAFIS\#4082-2016021510499450v2). Experiments were conducted in strict accordance with the recommendations and guidelines of the European Union (Directive2010/63/EU) and the French National Committee (Décret 2013-118). All efforts were made to improve animal welfare and minimize animals suffering.

Drugs and treatments. Mefloquine (MEF; Sigma-Aldrich), meclofenamic acid (MFA; Sigma-Aldrich) and flecainide acetate (FLE; Sigma-Aldrich) were freshly prepared in $0.9 \% \mathrm{NaCl}$ with $2 \% \mathrm{DMSO}$ and administered intraperitoneally (i.p.) at the same dose of $1 \mathrm{mg} / \mathrm{kg}$ in a volume of $5 \mathrm{~mL} / \mathrm{kg}, 2.5 \mathrm{~h}$ before imaging. Control animals received the vehicle only under the very same conditions.

Preparation and stereotaxic injection of lentiviral vectors. MOKOLA pseudotyped lentiviral vector (LV) encoding a shRNA directed against $\mathrm{Cx} 43$ (passenger-loop-guide strand: AACAGTCTGCCTTTCGCTGTATAGTGAAGCCACAGATGTA-TACAGCGAAAGGCAGACTGTT) or GFP (passenger-loop-guide strand: GCAAGCTGACCCTGAAGTTCAT-CTGTGAAGCCACAGATGGG-ATGAACTTCAGGGTCAGCTTGC) mRNA (control vector; shCTRL-LV) within astrocytes were generated as previously described ${ }^{17,18}$. For these recombinant viruses, the viral envelope ensures the specific tropism towards astrocytes. In addition, a detargeting strategy using miR9*T and miR124T was set up to eliminate any possible residual expression in neuronal cells ${ }^{17-19}$.

Mice were anesthetized with ketamine $75 \mathrm{mg} / \mathrm{kg}$ i.p. and xylazine $10 \mathrm{mg} / \mathrm{kg}$ i.p. LV were injected using a 34-gauge blunt-tip needle connected to a Hamilton syringe (Reno) with a polyethylene catheter. Dilutions of viral preparations in PBS containing 1\% bovine serum albumin (BSA) were made so as to reach a final concentration of $100,000 \mathrm{ng} \mathrm{p} 24 \mu \mathrm{L}^{-1}$ in the suspensions for injections. Mice received unilateral injections of $1 \mu \mathrm{L} \mathrm{LV}$ suspension at a rate of $0.1 \mu \mathrm{L} / \mathrm{min}$ at three different sites into the hippocampus. ShCx43-LV was injected on one side (either right or left, randomly chosen) and shCTRL-LV was injected on the contralateral side (left or right, respectively) in each mouse. The stereotaxic coordinates for these three unilateral injections were respectively: site $1=\mathrm{AP}$, $-1.94 ; \mathrm{L}, \pm 2.00$ and $\mathrm{V},-1.50$; site $2=\mathrm{AP},-2.46 ; \mathrm{L}, \pm 2.50$ and $\mathrm{V},-2.60$; site $3=\mathrm{AP},-3.0 ; \mathrm{L}, \pm 2.7$ and $\mathrm{V},-3.5$ (in $\mathrm{mm}$ from bregma ${ }^{20}$ ). At the end of each injection, the needle was left in place for $5 \mathrm{~min}$ before being slowly removed. At the end of the injection procedure, the skin was sutured with 4-0 silk thread (Mersilkä, Ethicon Inc.) and mice were allowed to recover under a heating light before returning in their home cage.

MRI acquisitions. The MEMRI technique is based on the paramagnetic properties of $\mathrm{Mn}^{2+}$ which induce a marked reduction of the longitudinal $\mathrm{T}_{1}$ relaxation time during MRI. MRI signal acquisitions were performed on horizontal bore small animal scanners (Bruker BioSpin, Ettlingen, Germany) operating at $7 \mathrm{~T}$ and 11.7 T using a 4-channel mouse brain array coil and a two-channel mouse brain cryoprobe, respectively. The mice were anesthetized with $0.5-1.0 \%$ isoflurane in air throughout the experiments. The respiration rate was monitored and the body temperature was maintained at $37^{\circ} \mathrm{C}$ using an MR-compatible, feedback-controlled air heating system (model 1025; SA Instruments, NY) or circulating hot water system. A capillary filled with $100 \mu \mathrm{M} \mathrm{MnCl}_{2}$ (in distilled water) was placed on the side of the head, inside the radiofrequency (RF) coil, and was used as reference for $\mathrm{T}_{1}$-weighted signal intensity normalization.

The $\mathrm{T}_{1}$-weighted images were acquired using a $3 \mathrm{D}$ rapid acquisition with relaxation enhancement (RARE) sequence: RARE factor 4 , repetition time/echo time $=250 \mathrm{~ms} / 6 \mathrm{~ms}$, 4 averages. For $\mathrm{T}_{1}$ relaxation time measurements, we used a true Fast Imaging with Steady-state free Precession (trueFISP) sequence with the following acquisition parameters: repetition time/echo time $=3.66 / 1.83 \mathrm{~ms}$, flip angle $=10$, inversion time $=80 \mathrm{~ms}$, number of inversion times $=60,2$ averages. The spatial resolutions and acquisition times for the two sequences on the two MRI systems are listed in Table 1.

Data processing. The position of the brain image was co-registered to a template using SPM8 software (Welcome Trust Center for Neuroimaging, UK). The template image was co-registered to mouse brain atlas ${ }^{20}$. $\mathrm{T}_{1}$-weighted signals were normalized using the signal intensity from the reference tube. $\mathrm{T}_{1}$ maps were calculated as described previously ${ }^{21}$. Eighteen regions of interest (ROIs) were defined using the mouse brain atlas. The averaged $\mathrm{T}_{1}$-weighted signals and $\mathrm{T}_{1}$ values were calculated using an in-house written Matlab program. To compensate for differences in coil sensitivity between left and right channels, we calculated the signal intensities in ROIs drawn in the left and right cerebral cortex, including the somatosensory cortex and insular cortex (ROI cortex_left $_{\text {. }}$ 
A

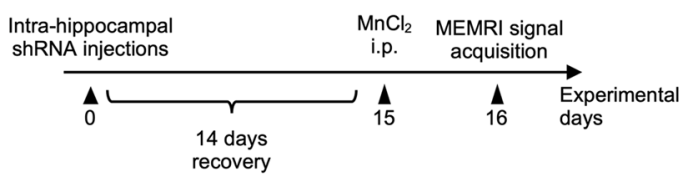

B Intra-hippocampal shRNA injections

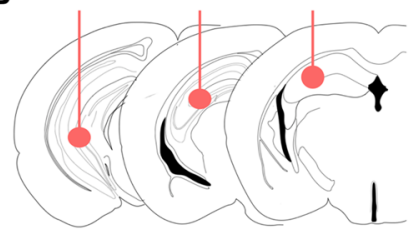

C

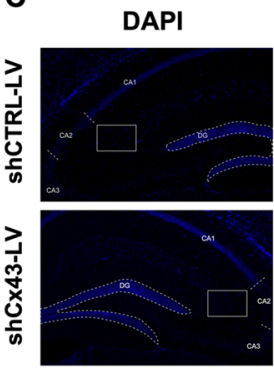

Cx43

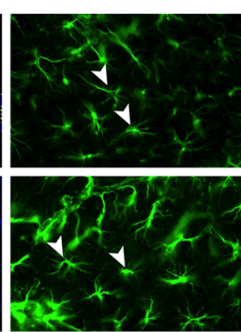

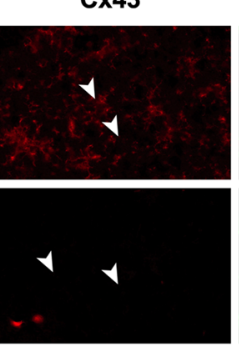

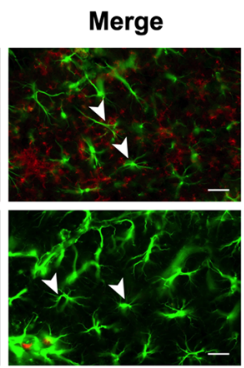

D

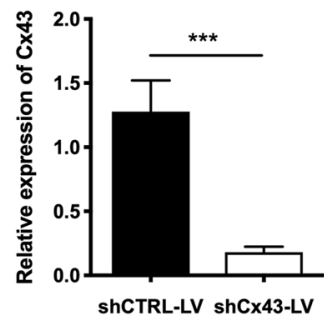

E
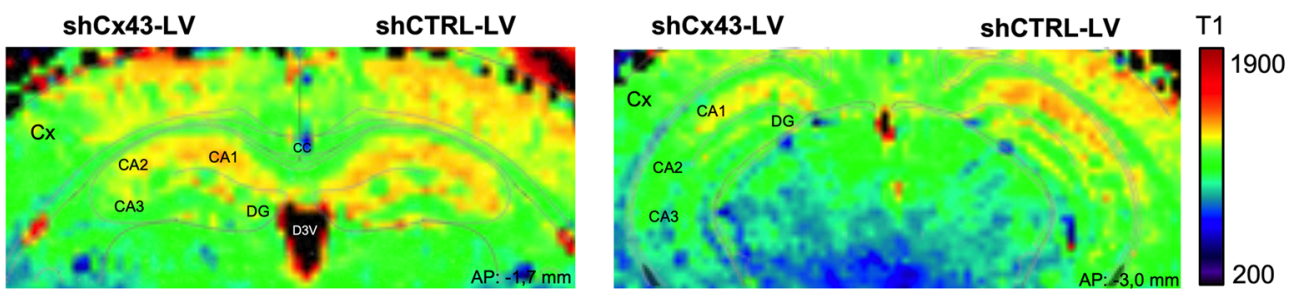

$\mathbf{F}$
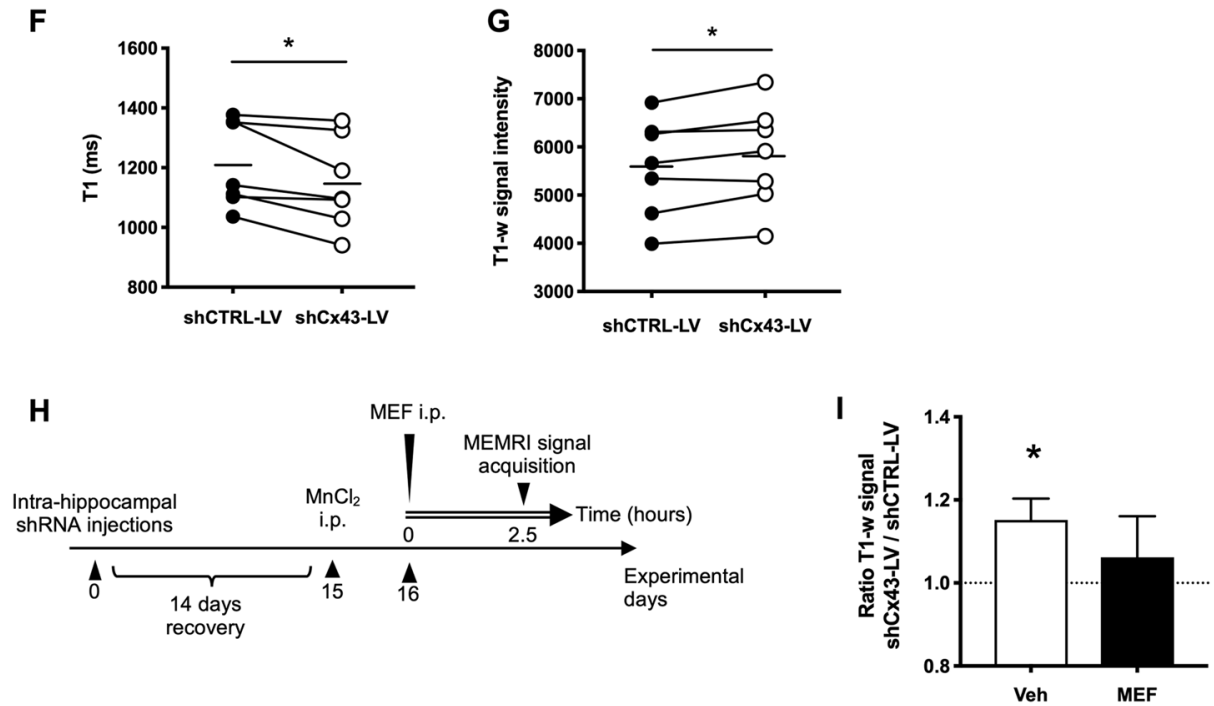

Figure 1. MEMRI signal responses to selective Cx43 knockdown expression in the mouse hippocampus. (A) Experimental timeline used for the experiments shown in E, G and Table 2. Mice received intra-hippocampal shRNA injections and were allowed to recover for 14 days. On day $15, \mathrm{MnCl}_{2}(50 \mathrm{mg} / \mathrm{kg})$ was delivered i.p. and MEMRI signal acquisition was performed $24 \mathrm{~h}$ later. (B) shRNA stereotaxic injection procedure. Mice received unilateral injections of LV suspension at three different sites into the hippocampus. ShCx43-LV was injected on one side and shCTRL-LV on the contralateral side (C) Representative fluorescence microscopy images of hippocampal shCTRL-LV- and shCx43-LV-injected sides showing DAPI (blue), GFAP + cells (green) and Cx43 expression (red). Arrow heads point at typical double-labeled GFAP+/Cx43+ astrocytes (top; shCTRL-LV) or highlight the dramatic decrease in the hippocampal expression of Cx43 (bottom; shCx43-LV injected hippocampus). Scale bar: $20 \mu \mathrm{m}$. (D) Relative expression of Cx43 in the hippocampus on the shCTRL-LV versus the shCx43-LV-injected side. ***p $<0.001$, Mann-Whitney test. Each bar is the mean \pm SEM of 9 independent determinations. (E) Typical MEMRI signal responses maps (T1) at hippocampal level (AP $=-1.7 \mathrm{~mm}$ and $\left.-3.0 \mathrm{~mm}^{20}\right)$ in a mouse injected with shCx43-LV on one side and shCTRL-LV on the other side. Cx, Cortex; CA1, 2, 3, respective regions of cornu ammonis; DG, Dentate Gyrus; D3V, Dorsal 3rd ventricle. (F) Respective $\mathrm{T}_{1}$ (in $\mathrm{ms}$ ) and $(\mathbf{G}) \mathrm{T}_{1}$-weighted signal intensity values in the hippocampus on shCTRL-LV and shCx43-LV injected side. MR acquisitions were performed on a 11.7 T Bruker imaging system. Significant differences in both parameter values were observed between the two sides; ${ }^{*} \mathrm{p}<0.05$, paired $t$ test. (H) Experimental timeline 
used for the experiments shown in I and Table 3. Mice received intra-hippocampal shRNA injections and were allowed to recover for 14 days. On day 15 , mice received $\mathrm{MnCl}_{2}(50 \mathrm{mg} / \mathrm{kg}$; i.p.) then $\mathrm{MEF}$ ( $1 \mathrm{mg} / \mathrm{kg}$; i.p.) or vehicle $24 \mathrm{~h}$ later. MEMRI signal acquisition was performed $2.5 \mathrm{~h}$ after the MEF administration. (I) Data are presented as mean \pm SEM of the ratio shCx43-LV/shCTRL-LV of MEMRI signal intensity in the hippocampus $2.5 \mathrm{~h}$ after i.p. administration of MEF or its vehicle. ${ }^{*} \mathrm{p}<0.05$, one sample $t$ test compared to the hypothetical value $1, \mathrm{n}=6$ in each group.

$\left.\mathrm{ROI}_{\text {cortex_right }}\right)$. The averaged T1-weighted signal intensity on the left side $\left(\mathrm{SI}_{\text {left }}\right)$ was then corrected following the equation:

$$
S I_{\text {left_corrected }}=S I_{\text {left }} \frac{S I_{\text {cortex_right }}}{S I_{\text {cortex_left }}}
$$

Immunohistochemistry. After MRI acquisitions, mice were deeply anesthetized and transcardially perfused with $4 \%$ paraformaldehyde in phosphate-buffered saline (PBS). Series of one in twelve $30 \mu \mathrm{m}$ thick coronal sections were incubated in a solution of rabbit anti-GFAP (1:500, Agilent technologies) and mouse anti-Cx43 (1:250, BD transduction) overnight at $4^{\circ} \mathrm{C}$. Sections at the very injections sites and displaying signs of lesions were discarded. After several rinses in PBS containing 0.25\% Triton-X100 (PBST), sections were incubated for $2 \mathrm{~h}$ at room temperature in a mixture of Alexa Fluor ${ }^{\circledR} 488$-conjugated highly cross-adsorbed donkey anti-rabbit and Alexa Fluor ${ }^{\circledR}$ 555-conjugated donkey anti-mouse (all at 1:500; Life technologies, ThermoFisher) antibodies in PBST supplemented with $10 \%$ normal donkey serum and $0.1 \%$ BSA. Sections were finally rinsed extensively and mounted onto slides, coverslipped using Mowiol ${ }^{\circledR}$ and stored at $4{ }^{\circ} \mathrm{C}$.

On the other hand, coronal sections at hippocampal level from mice injected locally with shCx43-LV on one side and shCTRL-LV on the other side were labeled with the nucleic acid dye $4^{\prime} 6$-diamidino-2-phenylindole (DAPI) to visualize all cell nuclei and verify that recombinant lentiviruses were devoid of any neurotoxic effect.

Quantification of $\mathrm{Cx} 43$ deletion. Cx43-immunolabeled hippocampi were pictured for each injection site using an Olympus BX-51 microscope equipped with Mercator Software (Explora Nova, France). The dentate gyrus area was framed at $\mathrm{x} 40$ magnification lens for each injection site. Quantification of Cx43-labeled sections was conducted with the FIJI software (ImageJ 2, NIH). Dentate gyri of the three injection sites sections were defined as the region of interest and a square $(800 \times 800 \mathrm{px})$ was delineated on each injection site to measure the mean gray value. Value intensity of $\mathrm{Cx} 43$ gray label was normalized to the GFAP gray label value in the very same area. Results are presented as the mean \pm SEM of the three injection sites gray value.

Experimental design and statistical analyses. Animals were divided into three sets of experiments as described in the experiment timelines (Figs. 1A,H and 2A): LV intra-hippocampal injections only (Set A, n=7); LV intra-hippocampal injections and vehicle or MEF i.p. administration (Set $B, n=11$ ); vehicle, MEF, MFA or FLE i.p. administration (Set $\mathrm{C}, \mathrm{n}=24$ ). In set $\mathrm{A}$, animals were injected with $\mathrm{MnCl}_{2} 50 \mathrm{mg} / \mathrm{kg}$ (in distilled water) i.p. two weeks after LV injections, and MRI acquisitions (see below) were performed $24 \mathrm{~h}$ later. In set $\mathrm{B}$, mice were injected with $\mathrm{LV}$ and $\mathrm{MnCl}_{2}$ as in set A, then received $\mathrm{MEF}$ treatment $24 \mathrm{~h}$ after i.p. $\mathrm{MnCl}_{2}$ and $2.5 \mathrm{~h}$ before MRI acquisitions. In set C, mice received only MEF, MFA or FLE i.p. $24 \mathrm{~h}$ after i.p. $\mathrm{MnCl}_{2}$ and $2.5 \mathrm{~h}$ before MRI acquisitions.

Statistical analyses were performed using GraphPad Prism version 7.0c. Data were analyzed using Mann-Whitney non-parametric test, paired $t$ test or one sample $t$ test compared to the hypothetical value 1 or 100 as appropriate. In each experiment, a level of $\mathrm{p}<0.05$ was accepted as evidence for a statistically significant effect $(* \mathrm{p}<0.05 ; * * \mathrm{p}<0.01 ; * * \mathrm{*}<0.001)$. Changes in MEMRI signal in response to the Cx43 expression knock-down and/or drugs administration are expressed as means \pm SEM of percent of control. All other data are expressed as mean \pm SEM. For analysis of MEMRI signal responses to drugs administration, quantification was made in six brain areas selected on the basis of their different response patterns.

\section{Results}

Selective silencing of astrocyte Cx43 expression in the mouse hippocampus. Recombinant LV with a glial tropism and expressing shRNA against $\mathrm{Cx} 43$ (shCx43-LV) were stereotaxically injected into one hippocampus to generate mice with a unilateral specific inactivation of astroglial Cx43 in this region ${ }^{19}$. Recombinant LV expressing shRNA against GFP (shCTRL-LV) were injected into the contralateral hippocampus as control (Fig. 1B,C). DAPI labeling showed that the cell density in both hippocampi did not differ from each other and from that determined in control mice (not shown). The characteristic punctate Cx43-like immunoreactivity in shCTRL-LV injected hippocampus was shown to co-localize with GFAP-immunoreactive astrocyte (Fig. 1C). Quantification of immunostained brain sections confirmed that mice displayed a dramatic decrease in the hippocampal expression of $\mathrm{Cx} 43$ on their shCx43-LV-injected side relative to their shCTRL-LV-injected contralateral side (approx. $-85 \%$; $\mathrm{p}<0.001$; Mann-Whitney test; Fig. 1C,D).

MEMRI signal responses to selective Cx43 knockdown expression in the mouse hippocampus. $\mathrm{Mn}^{2+}$ accumulation, because of its ability to shorten the longitudinal relaxation time $\left(\mathrm{T}_{1}\right)$ of the proton, produces hyperintense regions on $\mathrm{T}_{1}$-weighted images. To assess the impact of $\mathrm{Cx} 43$ knockdown on $\mathrm{Mn}^{2+}$ accumulation, we applied the MEMRI technique in shCx43-LV-injected mice which received $\mathrm{MnCl}_{2}$ via the i.p. route $24 \mathrm{~h}$ before 
A

\begin{tabular}{|c|c|c|}
\hline$\underset{\text { i.p. }}{\mathrm{MnCl}_{2}}$ & $\begin{array}{l}\text { Treatment } \\
\text { i.p. }\end{array}$ & $\begin{array}{l}\text { MEMRI signal } \\
\text { acquisition }\end{array}$ \\
\hline 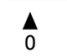 & $\underset{24}{\boldsymbol{A}}$ & $\frac{\Delta}{26.5}$ \\
\hline
\end{tabular}

B

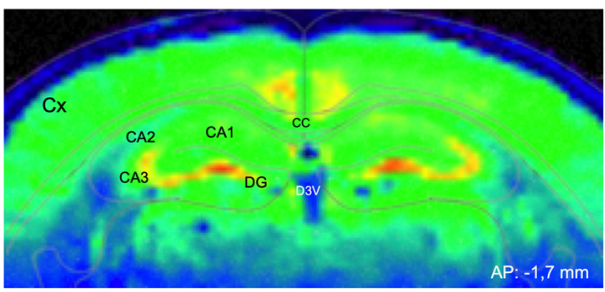

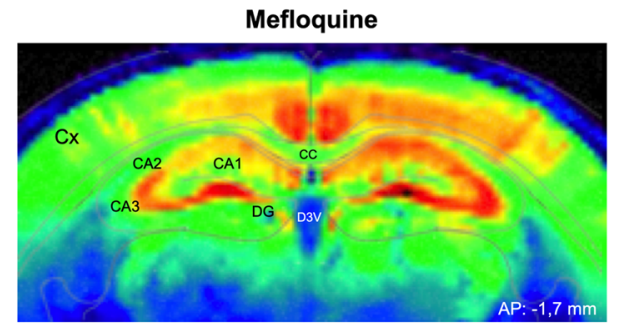

SI

C

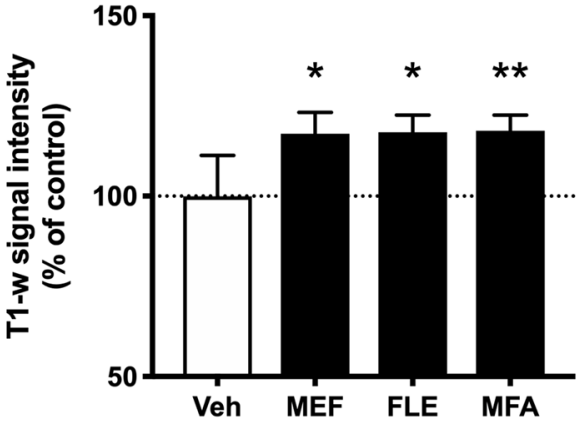

Figure 2. Effects of acute treatment with mefloquine (MEF), flecainide (FLE) or meclofenamic acid (MFA) versus vehicle (Veh) on MEMRI signal intensity in the mouse hippocampus. (A) Experimental timeline used for the experiments shown in $\mathrm{B}$ and C. Mice were injected with $\mathrm{MnCl}_{2}(50 \mathrm{mg} / \mathrm{kg}$; i.p.) then received connexin blocker (MEF, FLE or MFA, all at $1 \mathrm{mg} / \mathrm{kg}$ i.p.) or vehicle $24 \mathrm{~h}$ later. MEMRI signal acquisition was performed $2.5 \mathrm{~h}$ after the latter injection. (B) $\mathrm{T}_{1}$-weighted signal intensity maps in the hippocampus $\left(\mathrm{AP}=-1.7 \mathrm{~mm}^{20}\right)$ after vehicle or mefloquine administration. (C) Mice pretreated with $\mathrm{MnCl}_{2}$ were subjected to MEMRI $2.5 \mathrm{~h}$ after administration of each drug. MEMRI signal intensity in the hippocampus was compared to the respective value in vehicle-treated control mice. Data are presented as mean \pm SEM. Cx, Cortex; CA1, 2, 3, respective regions of cornu ammonis; DG, Dentate Gyrus; D3V, Dorsal 3rd ventricle. * $\mathrm{p}<0.05$, ** $<0.01, \mathrm{n}=6$ in each group.

the MEMRI acquisition. Figure 1E shows coronal T1 images of one representative mouse brain $(-1.7 \mathrm{~mm}$ from bregma, left panel; $-3.00 \mathrm{~mm}$ from bregma, right panel) $24 \mathrm{~h}$ after $\mathrm{MnCl}_{2}$ administration. A clear-cut decrease in MEMRI signal response appeared in the shCx43-LV injected hippocampus in comparison with the contralateral shCTRL-LV injected side (Fig. 1F). Quantification of images acquired with $7 \mathrm{~T}$ and 11.7 T MRI devices showed that $\mathrm{T}_{1}$ value for the hippocampus was significantly lower on the shCx43-LV injected side compared to the contralateral shCTRL-LV injected side $(-1.42 \pm 0.53 \%$ and $-5.31 \pm 1.65 \%$ with $7 \mathrm{~T}$ and $11.7 \mathrm{~T}$ MR imaging systems, respectively, $\mathrm{p}<0.05$; paired $t$ test, Table 2 ). On the other hand, a significant increase of the $\mathrm{T}_{1}$-weighted signal intensity was observed on the shCx43-LV injected side in comparison with the contralateral shCTRL-LV injected side $(+15.20 \pm 5.16 \%$ and $+3.91 \pm 1.24 \%$, with $7 \mathrm{~T}$ and $11.7 \mathrm{~T}$ MR imaging systems, respectively; $\mathrm{p}<0.05$; paired $t$ test) (Fig. 1G).

Quantification of MEMRI signal responses $\left(\mathrm{T}_{1}, \mathrm{~T}_{1}-\mathrm{w}\right)$ in 17 supplementary brain regions from mice injected with recombinant LV showed that no significant difference between the shCx43-LV injected side and the contralateral shCTRL-LV injected side was observed outside the hippocampus (Table 2). Thus, regardless the device on which the data were acquired: $7 \mathrm{~T}$ or $11.7 \mathrm{~T}$, significant differences in the $\mathrm{T}_{1}$ relaxation time and the $\mathrm{T}_{1}$-weighted signal intensity were noted only at the hippocampal level (Table 2).

Mefloquine treatment normalized interhemispheric differences in MEMRI signal responses in shCx43-LV injected mice. Mice with unilateral selective silencing of Cx43 expression in hippocampal astrocytes received $\mathrm{MnCl}_{2}$ i.p. injection two weeks after intra-hippocampal $\mathrm{LV}$ administration, and were treated $24 \mathrm{~h}$ later with MEF or vehicle i.p. $2.5 \mathrm{~h}$ before MEMRI signal acquisition (Fig. $1 \mathrm{H}$ ). The ratio shCx43/shCTRL for $\mathrm{T}_{1}$-weighed intensity in the Cx43 knockdown hippocampus over that in the contralateral control hippocampus was compared to the theoretical value, i.e. 1, expected for equal values on both sides. In concordance with data obtained in the previous experimental series (Fig. $2 \mathrm{G}$ ), the mean $\mathrm{T}_{1}$-weighted ratio was significantly different from $1(\mathrm{n}=6 ; \mathrm{p}<0.05$; one-sample $t$ test) in unilateral $\mathrm{Cx} 43$ knockdown mice treated with the vehicle (Fig. 2I). In contrast, after MEF administration, no difference was found in hippocampal T1-weighted intensity between 


\begin{tabular}{|c|c|c|c|c|c|c|c|c|}
\hline \multirow[b]{3}{*}{ Region } & \multicolumn{4}{|c|}{ Signal changes for shCx43-LV vs. shCTRL-LV (\%) } & \multicolumn{4}{|c|}{ Statistics } \\
\hline & \multicolumn{2}{|c|}{$7 \mathrm{~T}$} & \multicolumn{2}{|l|}{$11.7 \mathrm{~T}$} & \multicolumn{2}{|c|}{$7 \mathrm{~T}$} & \multicolumn{2}{|l|}{$11.7 \mathrm{~T}$} \\
\hline & $\mathrm{T}_{1}$ & $T_{1}-w$ & $\mathrm{~T}_{1}$ & $T_{1}-w$ & $\mathrm{~T}_{1}$ & $T_{1}-w$ & $\mathrm{~T}_{1}$ & $T_{1}-\mathrm{w}$ \\
\hline Hip & $-1.42 \pm 0.53$ & $15.2 \pm 5.16$ & $-5.31 \pm 1.65$ & $3.91 \pm 1.24$ & $<0.05$ & $<0.05$ & $<0.05$ & $<0.05$ \\
\hline SC & $0.40 \pm 0.57$ & $9.32 \pm 6.58$ & $-2.61 \pm 4.91$ & $3.12 \pm 3.57$ & 0.52 & 0.32 & 0.53 & 0.40 \\
\hline Cpu & $-0.34 \pm 0.49$ & $10.3 \pm 6.99$ & $-3.08 \pm 4.89$ & $-0.69 \pm 4.19$ & 0.54 & 0.33 & 0.41 & 0.84 \\
\hline $\mathrm{DRa}$ & $1.09 \pm 1.56$ & $8.67 \pm 7.24$ & $1.13 \pm 5.09$ & $0.67 \pm 5.13$ & 0.56 & 0.54 & 0.95 & 0.91 \\
\hline Hyp & $0.59 \pm 1.62$ & $8.20 \pm 7.65$ & $-2.48 \pm 4.58$ & $3.59 \pm 5.21$ & 0.81 & 0.63 & 0.41 & 0.62 \\
\hline LC & $0.52 \pm 1.18$ & $10.7 \pm 6.20$ & $-2.10 \pm 3.68$ & $-1.01 \pm 4.13$ & 0.66 & 0.22 & 0.46 & 0.86 \\
\hline LPB & $-0.83 \pm 1.14$ & $13.7 \pm 7.04$ & $-6.56 \pm 5.57$ & $-2.00 \pm 4.47$ & 0.47 & 0.09 & 0.20 & 0.74 \\
\hline $\mathrm{MC}$ & $0.58 \pm 0.51$ & $9.34 \pm 6.57$ & $-4.30 \pm 3.81$ & $0.47 \pm 3.78$ & 0.33 & 0.37 & 0.28 & 0.82 \\
\hline $\mathrm{OB}$ & $-0.07 \pm 0.39$ & $9.61 \pm 8.10$ & $-5.88 \pm 5.16$ & $2.27 \pm 5.27$ & 0.76 & 0.60 & 0.26 & 0.86 \\
\hline $\mathrm{SN}$ & $-0.85 \pm 1.05$ & $14.2 \pm 6.74$ & $-5.93 \pm 5.96$ & $0.54 \pm 4.02$ & 0.46 & 0.13 & 0.28 & 0.77 \\
\hline ThN & $-0.23 \pm 0.54$ & $13.1 \pm 6.72$ & $-3.39 \pm 2.92$ & $-0.85 \pm 4.40$ & 0.68 & 0.21 & 0.23 & 0.86 \\
\hline OC & $-0.42 \pm 1.12$ & $9.85 \pm 7.68$ & $-3.98 \pm 5.06$ & $0.75 \pm 4.45$ & 0.72 & 0.45 & 0.39 & 0.96 \\
\hline $\operatorname{PrL}$ & $0.93 \pm 1.87$ & $7.59 \pm 9.92$ & $-2.36 \pm 4.76$ & $7.45 \pm 6.93$ & 0.70 & 0.68 & 0.51 & 0.48 \\
\hline $\mathrm{AC}$ & $-1.10 \pm 0.84$ & $13.4 \pm 12.9$ & $4.32 \pm 6.91$ & $6.60 \pm 9.03$ & 0.25 & 0.32 & 0.78 & 0.56 \\
\hline IC & $-0.16 \pm 0.64$ & $11.3 \pm 9.44$ & $-3.39 \pm 9.06$ & $4.15 \pm 2.13$ & 0.77 & 0.29 & 0.49 & 0.12 \\
\hline $\mathrm{Cg}$ & $-0.29 \pm 1.45$ & $7.77 \pm 9.06$ & $-3.97 \pm 3.83$ & $1.19 \pm 5.16$ & 0.81 & 0.60 & 0.33 & 0.82 \\
\hline $\mathrm{mPFC}$ & $0.56 \pm 1.10$ & $7.17 \pm 9.83$ & $-0.14 \pm 5.57$ & $4.34 \pm 6.28$ & 0.61 & 0.72 & 0.83 & 0.69 \\
\hline Sep & $-1.41 \pm 1.17$ & $11.4 \pm 8.20$ & $-7.57 \pm 7.73$ & $2.11 \pm 4.94$ & 0.31 & 0.44 & 0.28 & 0.75 \\
\hline
\end{tabular}

Table 2. Effects of astroglial Cx43 expression knockdown in the hippocampus on MEMRI signal in various brain regions. MEMRI signal response $\left(\mathrm{T}_{1}\right.$ and $\mathrm{T}_{1}-\mathrm{w}$, means $\left.\pm \mathrm{SEM}\right)$ in each brain region was compared in the shCx43-LV-injected side versus the shCTRL-LV-injected side in each mouse (paired $t$ test). Abbreviations: SC, Somatosensory cortex; Cpu, Caudate putamen; Dra, Dorsal raphe pallidus; Hip, Hippocampus; Hyp, Hypothalamus; LC, Locus coeruleus; LPB, Lateral parabrachial nucleus; MC, Motor cortex; OB, Olfactory bulb; SN, Substantia nigra; ThN, Thalamic nuclei; OC, Orbital cortex, PrL, Prelimbic cortex; AC, Auditory cortex; IC, Insular cortex; Cg, Cingulate cortex; mPFC, Medial prefrontal cortex; Sep, Septum.

\begin{tabular}{|l|l|l|l|l|}
\hline \multirow{2}{*}{ Region } & \multicolumn{2}{l}{ Ratio $\mathbf{T}_{\mathbf{1}}$-w } & \multicolumn{2}{l|}{ Statistics } \\
\cline { 2 - 5 } & \multicolumn{2}{|l}{ shCx43-LV/shCTRL-LV } & \multicolumn{2}{l|}{$\begin{array}{l}\text { shCX3-LV/ } \\
\text { shCTR-LV }\end{array}$} \\
\cline { 2 - 5 } & Veh & MEF & Veh & MEF \\
\hline Hip & $\mathbf{1 . 1 5} \pm \mathbf{0 . 0 5}$ & $\mathbf{1 . 0 6} \pm \mathbf{0 . 1 0}$ & $<\mathbf{0 . 0 5}$ & $\mathbf{0 . 5 6}$ \\
\hline SC & $1.09 \pm 0.07$ & $1.04 \pm 0.16$ & 0.22 & 0.79 \\
\hline Cpu & $1.10 \pm 0.07$ & $1.05 \pm 0.16$ & 0.20 & 0.75 \\
\hline DRa & $1.09 \pm 0.07$ & $1.08 \pm 0.19$ & 0.28 & 0.71 \\
\hline Hyp & $1.08 \pm 0.08$ & $1.09 \pm 0.20$ & 0.33 & 0.67 \\
\hline LC & $1.11 \pm 0.06$ & $1.06 \pm 0.13$ & 0.15 & 0.67 \\
\hline LPB & $1.14 \pm 0.07$ & $1.07 \pm 0.12$ & 0.11 & 0.58 \\
\hline MC & $1.09 \pm 0.07$ & $1.03 \pm 0.17$ & 0.21 & 0.85 \\
\hline OB & $1.10 \pm 0.08$ & $1.08 \pm 0.18$ & 0.29 & 0.68 \\
\hline SN & $1.14 \pm 0.07$ & $1.07 \pm 0.13$ & 0.090 & 0.60 \\
\hline ThN & $1.13 \pm 0.07$ & $1.07 \pm 0.16$ & 0.11 & 0.70 \\
\hline OC & $1.10 \pm 0.08$ & $1.08 \pm 0.19$ & 0.26 & 0.71 \\
\hline PrL & $1.08 \pm 0.10$ & $1.10 \pm 0.24$ & 0.48 & 0.70 \\
\hline AC & $1.13 \pm 0.13$ & $0.97 \pm 0.14$ & 0.35 & 0.86 \\
\hline IC & $1.11 \pm 0.09$ & $1.03 \pm 0.16$ & 0.29 & 0.88 \\
\hline Cg & $1.08 \pm 0.09$ & $1.07 \pm 0.21$ & 0.43 & 0.74 \\
\hline mPFC & $1.07 \pm 0.10$ & $1.08 \pm 0.22$ & 0.50 & 0.74 \\
\hline Sep & $1.11 \pm 0.08$ & $1.09 \pm 0.19$ & 0.22 & 0.66 \\
\hline
\end{tabular}

Table 3. Effects of mefloquine (MEF) treatment on MEMRI signal in various brain regions of mice with unilateral hippocampal Cx43 silencing. In both MEF- and vehicle (Veh)-treated mice, data are expressed as the ratio of MEMRI signal intensity in each brain area on the shCx43-LV-injected side over the shCTRL-LVinjected side. Statistical analyses were made by comparing each ratio (mean $\pm S E M$ ) with the hypothetical value 1. Abbreviations: SC, Somatosensory cortex; Cpu, Caudate putamen; Dra, Dorsal raphe pallidus; Hip, Hippocampus; Hyp, Hypothalamus; LC, Locus coeruleus; LPB, Lateral parabrachial nucleus; MC, Motor cortex; OB, Olfactory bulb; SN, Substantia nigra; ThN, Thalamic nuclei; OC, Orbital cortex, PrL, Prelimbic cortex; AC, Auditory cortex; IC, Insular cortex; Cg, Cingulate cortex; mPFC, Medial prefrontal cortex; Sep, Septum. 
the ShCx43-LV-injected side and the contralateral shCTRL-LV injected side $(\mathrm{n}=5 ; \mathrm{p}=0.562$; ns; one-sample $t$ test, Fig. 2I). Quantification of $\mathrm{T}_{1}$-weighted ratio of MEMRI signal intensity in Cx43-LV-injected side over that in the contralateral shCTRL-LV-injected side showed that none of the same 17 brain regions as those selected - in addition to the hippocampus - in the previous experimental series presented any significant difference in MEFversus vehicle-treated mice (Table 3).

MEMRI signal responses to Cx43 blockers in mouse hippocampus. We then investigated the effect, in vivo, of three pharmacological blockers of Cx43, MEF, MFA and FLE on MEMRI signal in the hippocampus of intact healthy mice (without intra-hippocampal LV injections) that had received $\mathrm{MnCl}_{2}$ via the i.p. route $24 \mathrm{~h}$ before (Fig. 2A). Figure $2 \mathrm{~B}$ showed the MEMRI signal intensity of selected coronal sections (both, $-1.7 \mathrm{~mm}$ from bregma) of a representative vehicle-treated mouse (left panel) in comparison with a MEF-treated mouse (right panel). These images show a clear-cut increase in hippocampal MEMRI signal after MEF treatment. Quantification of $\mathrm{T}_{1}$-w signal intensity in 6 mice in each group demonstrated that MEF-induced increase was statistically significant $(+18.0 \pm 6.0 \%, \mathrm{p}<0.05)$. As shown in Fig. $2 \mathrm{C}$, both FLE and MFA also significantly increased the $\mathrm{T}_{1}$ - $\mathrm{w}$ signal intensity in the mouse hippocampus, up to the same level as that found with MEF $($ FLE: $+18.0 \pm 4.7 \%, \mathrm{p}<0.05 ; \mathrm{MFA}:+18.0 \pm 4.3 \%, \mathrm{p}<0.01)$.

\section{Discussion}

Data from this study present a new application of MEMRI to investigate the functional activity of cerebral astroglial Cx43 in vivo, and to determine the effects of pharmacological blockers of astroglial Cxs in the mouse brain.

Neurons have long been considered as the primary performers of brain processes. However, during evolution, glia-to-neuron ratio significantly increases towards more and more adapted species. Among glial cells, astrocytes are closely connected to neurons and interactions between these two types of cells have largely been described ${ }^{22}$. Astrocytes have been successfully labeled with PET probes such as ${ }^{11} \mathrm{C}$-acetate ${ }^{23},{ }^{11} \mathrm{C}$-DED and ${ }^{11} \mathrm{C}$-Sch $2253366^{10}$, passive fluorescent dyes such as sulforhodamine $101^{11}$ or genetically encoded $\mathrm{Ca}^{2+}$ indicators (GECIs) ${ }^{12}$. However, neurons, as well as astrocytes, readily take up blood-born lactate ${ }^{24}$, and so far, the use of GECIs or dyes has been limited due the requirement of their local intracerebral application and the low penetrance depth of optical microscopy ${ }^{13}$. In vivo brain imaging of the interactions between astrocytes and neurons is hence still challenging.

The functional activity of astrocyte networks involves key transmembrane proteins called $\mathrm{Cxs}^{5}$. Eleven $\mathrm{Cx}$ isoforms have been detected in the brain, and, among them, astrocytes express Cx43 and Cx30 isoforms in adult brains. While Cx30 is abundantly present in the thalamus, Cx43 and Cx30 are expressed at similar levels in the hippocampus ${ }^{6,25}$. The coupling between astrocytes is significantly reduced in cells lacking Cx43, demonstrating that this isoform is a major support for direct intercellular communication in astrocytes, notably in the hippocampus ${ }^{6,26}$. Additionally, Cx43-mediated networks have been shown to play an important role in several neuronal modulation processes $^{2,6-8,27}$. In the present study, aimed at evaluating the role of Cx43 in Mn-based imaging, we silenced Cx43 expression in the hippocampus on one side and used the contralateral hippocampus as control. Unilateral Cx43 knockdown was achieved by intra-hippocampal injection of a lentiviral recombinant vector, shCx43-LV, pseudotyped with the MOKOLA envelop, and encoding a shRNA directed against Cx43 RNA within hippocampal astrocytes but not hippocampal stem cells or neurons ${ }^{17,18}$. Immunohistochemical controls fully confirmed that extinction of Cx43 expression occurred in astrocytes of the (randomly left or right) hippocampus injected with shCx43-LV. In contrast, $\mathrm{Cx} 43$ expression was preserved in the contralateral hippocampus injected with the control recombinant virus, shCTRL-LV, encoding non-relevant shRNA (shRNA against GFP). Interestingly, semi-quantitative estimates of DAPI labeling showed that none of these recombinant viruses had neurotoxic effects.

To compare the Mn-enhanced MR signal intensity in both sides of the hippocampus, as well as in other brain regions, $\mathrm{LV}$-injected mice were administered $\mathrm{MnCl}_{2}$ i.p. 24 hours before imaging, i.e. under time conditions allowing $\mathrm{Mn}^{2+}$ concentration and distribution to reach equilibrium in brain ${ }^{28}$. The $\mathrm{MnCl}_{2}$ dose $(50 \mathrm{mg} / \mathrm{kg}$ i.p. $)$ was chosen to avoid any neurological symptoms or neurotoxicity ${ }^{29}$. MEMRI analyses on both hippocampal sides indicated that $\mathrm{T}_{1}$ was significantly shortened when $\mathrm{Cx} 43$ expression was inhibited. $\mathrm{T}_{1}$-weighted signal confirmed this observation within the hippocampus. In contrast, MEMRI analyses in the other 17 brain regions examined did not reveal any difference between the shCx43-LV injected side and the contralateral shCTRL-LV injected side. These data indicate that manganese was more concentrated only in the hippocampus where Cx43 expression was silenced ${ }^{14,30}$. There are two possible explanations for this differential manganese accumulation between the two hippocampi: (i) $\mathrm{Mn}^{2+}$ accumulates more in astrocytes on the shCx43-LV injected side as a result of a reduced diffusion through deficient gap junctions or decreased release caused by the lower density of $\mathrm{Cx} 43$ hemichannels or (ii) $\mathrm{Mn}^{2+}$ accumulates more in neurons as a functional consequence of $\mathrm{Cx} 43$ downregulation in astrocytes. As a matter of fact, although $\mathrm{Mn}^{2+}$ accumulation specifically in astrocytes, neurons, or other cells cannot be determined with the presently available methods, our study reveals that down regulation of Cx43 selectively in hippocampal astrocytes leads to changes in the local MEMRI signal and provides a first in vivo validation of a functional role for astrocyte Cx43 using whole brain imaging. Noteworthy, similar results were obtained with 7 and 11.7 Tesla fields, widening the potential applications of MEMRI using different types of equipment. Validation in other brain areas, with different expression levels of $\mathrm{Cx} 43$, such as cortex or striatum ${ }^{31}$, might also be interesting, especially regarding regional molecular heterogeneity of hemichannels and gap junctions involved in astrocyte-neuron interactions ${ }^{32}$. The respective contribution of hemichannels or gap junctions in the transport of manganese remains an important issue, which is, however, complex to evaluate due to the lack of specific in vivo tools. Interestingly, using a cell line derived from normal adult human osteoblasts, Romanello et al. ${ }^{33}$ highlighted that hemichannels participated in $\mathrm{Mn}^{2+}$ uptake from the external medium. On the other hand, Niessen et al. ${ }^{34}$ demonstrated that $\mathrm{Mn}^{2+}$ had the ability to diffuse within monolayers of transfected HeLa cells expressing murine Cx26, Cx32 as well as Cx43. These two series of data support the idea that both hemichannels and gap junctions are involved in manganese transport within adjacent cytoplasms and/or between cytoplasm and extracellular space. 
Although in vitro studies on cultured astrocytes led Lu et al. ${ }^{35}$ to report that $\mathrm{Cx} 43$ expression could be affected by $\mathrm{Mn}^{2+}$, the cation concentration required for this effect, $125-1000 \mu \mathrm{M}$, was higher than that which was probably reached in brain under our in vivo conditions, i.e. $24 \mathrm{~h}$ after a unique injection of $\mathrm{MnCl}_{2}$ at $50 \mathrm{mg} / \mathrm{kg}$. With such conditions, available literature data on brain accumulation of peripherally injected $\mathrm{MnCl}_{2}{ }^{36,37}$ led us to estimate that $\mathrm{Mn}^{2+}$ did not exceed $50 \mu \mathrm{M}$ at the time of MEMRI signal acquisition. Even if direct measurements of brain Mn concentration will be needed to definitively exclude any possible alteration in $\mathrm{Cx} 43$ expression, it has to be emphasized that such alteration would occur equally in both hippocampi. Since our analyses consisted of comparing the hippocampus on the shCx43-LV-injected side with that on the contralateral shCTRL-LV-injected side, any global change in brain $\mathrm{Cx} 43$ caused by $\mathrm{Mn}^{2+}$ would not challenge the meaning of our data.

The next step for further investigations on the in vivo functional role of astrocyte Cx43 would be to quantify MEMRI signal in response to $\mathrm{Cx} 43$ up regulation specifically in astrocytes in the mouse hippocampus. However, to date, recombinant $\mathrm{LV}$ for $\mathrm{Cx} 43$ overexpression specifically in astrocytes are still under development and are not yet available for such studies.

It is well established that MEF inhibits Cx43-mediated functions, through gap junctions ${ }^{38,39}$ and hemichannels ${ }^{40}$, potentially via loop gating ${ }^{41}$. Interestingly, in naive healthy mice, MEF significantly increased the MEMRI signal in the hippocampus, supporting the idea that in Cx43 knock-down animals, MEF concealed the disequilibrium in MEMRI signal between both hippocampi by increasing the signal on the shCTRL-LV injected side up to that already achieved by $\mathrm{Cx} 43$ knockdown on the shCx43-LV injected side. Interestingly, the other two drugs tested, FLE and MFA, which are known to cross the brain-blood barrier and exert clear-cut inhibitory effects on Cx43 (but through still uncharacterized mechanisms) ${ }^{42,43}$, similarly increased MEMRI signal in the hippocampus in naive healthy mice, further supporting the idea that MEMRI signal in this brain region is related to Cx43 function.

Meanwhile, targeting Cxs, using in particular MEF, FLE and MFA, can result in changes in the properties of CNS drugs, in terms of pharmacological profiles ${ }^{40,42}$. Such techniques as MEMRI may help determining the mechanism of action of pharmacological combinations of CNS drugs with Cx targeted compounds, notably in terms of localization of $\mathrm{Cx}$ modulations in brain. Indeed, $\mathrm{Cx}$ expression displays regional heterogeneity within and between brain regions 9 . Furthermore, $\mathrm{Cx}$ functions can also be differentially regulated by local and region specific modulators such as neurotransmitters ${ }^{9}$. Interestingly, in our study, MEF restored equilibrium of the $\mathrm{T}_{1}$ relaxation time of MEMRI signal between both hippocampi in mice injected with shCx43-LV on one side and shCTRL-LV on the contralateral side, which provided a direct demonstration of an in vivo action of MEF on Cx43-mediated function in brain.

MEMRI has already been investigated in humans, notably with the use of FDA-approved mangafodipir, a $\mathrm{Mn}^{2+}$ chelate with the ligand fodipir ${ }^{44}$. Translational application of the present study, possibly with the use of mangafodipir, might provide new insights into neuron-astrocyte interactions in clinical trials and constitute a new tool for pharmacological evaluation of new modulators of those interactions. Additionally, as Cx43 expression is altered in neurodegenerative disorders such as Alzheimer's disease ${ }^{5,45}$, Parkinson's disease, Huntington's disease, amyotrophic lateral sclerosis ${ }^{46,47}$ and multiple sclerosis ${ }^{5,48}$, as well as in psychiatric disorders such as depression $^{18,49}$, Cx43 function evaluation using MEMRI might also be interesting for further pathophysiological investigations of those disorders. The diversity of $\mathrm{Cx}$ isoforms in the brains combined with their diverse functions should be further investigated to fully understand their roles in the brain.

Significance statement. Astrocytes are key non-neuronal cells implicated in many brain functions. These glial cells are organized in functional networks, mostly through connexin 43 (Cx43)-mediated gap junction channels. Astroglial networks are engaged in fine neuronal tuning and have recently been considered as emerging new therapeutic targets in CNS disorders. However, comprehensive understanding of the complex relationships between astrocytes and neurons in vivo is limited due to the lack of adequate imaging tools. Here, we demonstrate that manganese enhanced magnetic resonance imaging (MEMRI) is a promising technique for studying in vivo the function of astrocyte $\mathrm{Cx} 43$ and assessing the pharmacological profiles of $\mathrm{Cx} 43$ modulators.

Received: 23 May 2019; Accepted: 20 November 2019;

Published online: 04 December 2019

\section{References}

1. Rouach, N., Glowinski, J. \& Giaume, C. Activity-dependent neuronal control of gap-junctional communication in astrocytes. J Cell Biol 149, 1513-1526 (2000).

2. Dallerac, G. \& Rouach, N. Astrocytes as new targets to improve cognitive functions. Prog Neurobiol 144, 48-67, https://doi. org/10.1016/j.pneurobio.2016.01.003 (2016).

3. Pannasch, U. \& Rouach, N. Emerging role for astroglial networks in information processing: from synapse to behavior. Trends Neurosci 36, 405-417, https://doi.org/10.1016/j.tins.2013.04.004 (2013).

4. Araque, A., Parpura, V., Sanzgiri, R. P. \& Haydon, P. G. Tripartite synapses: glia, the unacknowledged partner. Trends Neurosci 22, 208-215 (1999)

5. Koulakoff, A., Mei, X., Orellana, J. A., Saez, J. C. \& Giaume, C. Glial connexin expression and function in the context of Alzheimer's disease. Biochim Biophys Acta 1818, 2048-2057, https://doi.org/10.1016/j.bbamem.2011.10.001 (2012).

6. Rouach, N., Koulakoff, A., Abudara, V., Willecke, K. \& Giaume, C. Astroglial metabolic networks sustain hippocampal synaptic transmission. Science 322, 1551-1555, https://doi.org/10.1126/science.1164022 (2008).

7. Escartin, C. \& Rouach, N. Astroglial networking contributes to neurometabolic coupling. Frontiers in neuroenergetics 5, 4, https:// doi.org/10.3389/fnene.2013.00004 (2013).

8. Chever, O., Dossi, E., Pannasch, U., Derangeon, M. \& Rouach, N. Astroglial networks promote neuronal coordination. Sci Signal 9, ra6, https://doi.org/10.1126/scisignal.aad3066 (2016).

9. Dallerac, G., Zapata, J. \& Rouach, N. Versatile control of synaptic circuits by astrocytes: where, when and how? Nat Rev Neurosci, 729-743, https://doi.org/10.1038/s41583-018-0080-6 (2018). 
10. Zimmer, E. R. et al. Tracking neuroinflammation in Alzheimer's disease: the role of positron emission tomography imaging. Journal of neuroinflammation 11, 120, https://doi.org/10.1186/1742-2094-11-120 (2014).

11. Appaix, F. et al. Specific in vivo staining of astrocytes in the whole brain after intravenous injection of sulforhodamine dyes. PLoS One 7, e35169, https://doi.org/10.1371/journal.pone.0035169 (2012).

12. Gee, J. M. et al. Imaging activity in astrocytes and neurons with genetically encoded calcium indicators following in utero electroporation. Front Mol Neurosci 8, 10, https://doi.org/10.3389/fnmol.2015.00010 (2015).

13. Perez-Alvarez, A., Araque, A. \& Martin, E. D. Confocal microscopy for astrocyte in vivo imaging: Recycle and reuse in microscopy. Front Cell Neurosci 7, 51, https://doi.org/10.3389/fncel.2013.00051 (2013).

14. Silva, A. C., Lee, J. H., Aoki, I. \& Koretsky, A. P. Manganese-enhanced magnetic resonance imaging (MEMRI): methodological and practical considerations. NMR Biomed 17, 532-543, https://doi.org/10.1002/nbm.945 (2004).

15. Wedler, F. C. \& Denman, R. B. Glutamine synthetase: the major Mn(II) enzyme in mammalian brain. Curr Top Cell Regul 24, 153-169 (1984)

16. Bade, A. N. et al. Improved visualization of neuronal injury following glial activation by manganese enhanced MRI. J Neuroimmune Pharmacol 8, 1027-1036, https://doi.org/10.1007/s11481-013-9475-3 (2013).

17. Colin, A. et al. Engineered lentiviral vector targeting astrocytes in vivo. Glia 57, 667-679, https://doi.org/10.1002/glia.20795 (2009).

18. Quesseveur, G. et al. Attenuated Levels of Hippocampal Connexin 43 and its Phosphorylation Correlate with Antidepressant- and Anxiolytic-Like Activities in Mice. Front Cell Neurosci 9, 490, https://doi.org/10.3389/fncel.2015.00490 (2015).

19. Merienne, N. et al. Gene transfer engineering for astrocyte-specific silencing in the CNS. Gene Ther 22, 830-839, https://doi. org/10.1038/gt.2015.54 (2015).

20. Franklin, K. B. J. \& Paxinos, G. The mouse brain in stereotaxic coordinates. 3rd ed./Keith B. J. Franklin, George Paxinos. edn, (Elsevier, 2007).

21. Schmitt, P. et al. Inversion recovery TrueFISP: quantification of T(1), T(2), and spin density. Magn Reson Med 51, 661-667, 10.1002/ mrm.20058 (2004).

22. Petit, J. M. \& Magistretti, P. J. Regulation of neuron-astrocyte metabolic coupling across the sleep-wake cycle. Neuroscience 323, 135-156, https://doi.org/10.1016/j.neuroscience.2015.12.007 (2016).

23. Wyss, M. T., Magistretti, P. J., Buck, A. \& Weber, B. Labeled acetate as a marker of astrocytic metabolism. J Cereb Blood Flow Metab 31, 1668-1674, https://doi.org/10.1038/jcbfm.2011.84 (2011).

24. Machler, P. et al. In Vivo Evidence for a Lactate Gradient from Astrocytes to Neurons. Cell Metab 23, 94-102, https://doi. org/10.1016/j.cmet.2015.10.010 (2016).

25. Gosejacob, D. et al. Role of astroglial connexin30 in hippocampal gap junction coupling. Glia 59, 511-519, https://doi.org/10.1002/ glia.21120 (2011)

26. Roux, L., Benchenane, K., Rothstein, J. D., Bonvento, G. \& Giaume, C. Plasticity of astroglial networks in olfactory glomeruli. Proc Natl Acad Sci USA 108, 18442-18446, https://doi.org/10.1073/pnas.1107386108 (2011).

27. Pannasch, U. et al. Astroglial networks scale synaptic activity and plasticity. Proc Natl Acad Sci USA 108, 8467-8472, https://doi. org/10.1073/pnas.1016650108 (2011).

28. Makris, A. P., Rush, C. R., Frederich, R. C. \& Kelly, T. H. Wake-promoting agents with different mechanisms of action: comparison of effects of modafinil and amphetamine on food intake and cardiovascular activity. Appetite 42, 185-195, https://doi.org/10.1016/j. appet.2003.11.003 (2004).

29. Galosi, R. et al. Identifying non-toxic doses of manganese for manganese-enhanced magnetic resonance imaging to map brain areas activated by operant behavior in trained rats. Magn Reson Imaging 37, 122-133, https://doi.org/10.1016/j.mri.2016.11.017 (2017).

30. Takeda, A. Manganese action in brain function. Brain Res Brain Res Rev 41, 79-87 (2003).

31. Nagy, J. I., Patel, D., Ochalski, P. A. \& Stelmack, G. L. Connexin30 in rodent, cat and human brain: selective expression in gray matter astrocytes, co-localization with connexin43 at gap junctions and late developmental appearance. Neuroscience 88, 447-468 (1999).

32. Hu, X., Yuan, Y., Wang, D. \& Su, Z. Heterogeneous astrocytes: Active players in CNS. Brain Res Bull 125, 1-18, https://doi. org/10.1016/j.brainresbull.2016.03.017 (2016).

33. Romanello, M., Veronesi, V. \& D’Andrea, P. Mechanosensitivity and intercellular communication in HOBIT osteoblastic cells: a possible role for gap junction hemichannels. Biorheology 40, 119-121 (2003).

34. Niessen, H., Harz, H., Bedner, P., Kramer, K. \& Willecke, K. Selective permeability of different connexin channels to the second messenger inositol 1,4,5-trisphosphate. J Cell Sci 113(Pt 8), 1365-1372 (2000).

35. Lu, C. et al. Involvement of gap junctions in astrocyte impairment induced by manganese exposure. Brain Res Bull 140, 107-113, https://doi.org/10.1016/j.brainresbull.2018.04.009 (2018).

36. Sotogaku, N., Oku, N. \& Takeda, A. Manganese concentration in mouse brain after intravenous injection. J Neurosci Res 61, 350-356, doi:10.1002/1097-4547(20000801)61:3<350::AID-JNR13>3.0.CO;2-4 (2000).

37. Roels, $\mathrm{H}$. et al. Influence of the route of administration and the chemical form $(\mathrm{MnCl} 2, \mathrm{MnO} 2)$ on the absorption and cerebral distribution of manganese in rats. Arch Toxicol 71, 223-230, https://doi.org/10.1007/s002040050380 (1997).

38. Picoli, C. et al. Human connexin channel specificity of classical and new gap junction inhibitors. J Biomol Screen 17, 1339-1347, https://doi.org/10.1177/1087057112452594 (2012).

39. Cruikshank, S. J. et al. Potent block of Cx36 and Cx50 gap junction channels by mefloquine. Proc Natl Acad Sci USA 101, 12364-12369 (2004).

40. Jeanson, T. et al. Potentiation of Amitriptyline Anti-Hyperalgesic-Like Action By Astroglial Connexin 43 Inhibition in Neuropathic Rats. Sci Rep 6, 38766, https://doi.org/10.1038/srep38766 (2016).

41. Verselis, V. K. \& Srinivas, M. Connexin channel modulators and their mechanisms of action. Neuropharmacology 75, 517-524, https://doi.org/10.1016/j.neuropharm.2013.03.020 (2013).

42. Duchêne, A. et al. Impact of Astroglial Connexins on Modafinil Pharmacological Properties. Sleep 39, 1283-1292 (2016).

43. Harks, E. G. et al. Fenamates: a novel class of reversible gap junction blockers. J Pharmacol Exp Ther 298, 1033-1041 (2001).

44. Cloyd, R., Vandsburger, M. \& Abisambra, J. F. A new opportunity for MEMRI. Aging (Albany NY) 9, 1855-1856, https://doi. org/10.18632/aging.101283 (2017).

45. Takeuchi, H. et al. Blockade of gap junction hemichannel suppresses disease progression in mouse models of amyotrophic lateral sclerosis and Alzheimer's disease. PLoS One 6, e21108, https://doi.org/10.1371/journal.pone.0021108 (2011).

46. Almad, A. A. et al. Connexin 43 in astrocytes contributes to motor neuron toxicity in amyotrophic lateral sclerosis. Glia 64, 1154-1169, https://doi.org/10.1002/glia.22989 (2016).

47. Cui, Y. et al. Extensive dysregulations of oligodendrocytic and astrocytic connexins are associated with disease progression in an amyotrophic lateral sclerosis mouse model. Journal of neuroinflammation 11, 42, https://doi.org/10.1186/1742-2094-11-42 (2014).

48. Masaki, K. et al. Connexin 43 astrocytopathy linked to rapidly progressive multiple sclerosis and neuromyelitis optica. PLoS One 8, e72919, https://doi.org/10.1371/journal.pone.0072919 (2013).

49. Nagy, C., Torres-Platas, S. G., Mechawar, N. \& Turecki, G. Repression of Astrocytic Connexins in Cortical and Subcortical Brain Regions and Prefrontal Enrichment of H3K9me3 in Depression and Suicide. Int J Neuropsychopharmacol 20, 50-57, https://doi. org/10.1093/ijnp/pyw071 (2016). 


\section{Acknowledgements}

The authors thank Boucif Djemai and Maria Rey for excellent technical support and general animal handling.

\section{Author contributions}

T.T. and L.C. performed the MRI experiments. T.T., B.P. and M.D. analyzed the data. M.D., M.C., B.P.G. and L.C. designed the experiments and managed the project. N.D. supervised the production of the viral vectors. M.C. wrote the first draft. M.D., T.T., A.D., B.P.G., N.R., M.H., F.M., L.C. and M.C. reviewed the article. All authors approved the final manuscript.

\section{Competing interests}

This work was supported by Theranexus Company. M.D., A.D., F.M. and M.C. are full-time employees of Theranexus; M.H. is a consultant for Theranexus. The other authors (T.T., B.P., B.P.G., N.D., N.R. and L.C.) declare no potential conflict of interest.

\section{Additional information}

Correspondence and requests for materials should be addressed to L.C. or M.C.

Reprints and permissions information is available at www.nature.com/reprints.

Publisher's note Springer Nature remains neutral with regard to jurisdictional claims in published maps and institutional affiliations.

(c) Open Access This article is licensed under a Creative Commons Attribution 4.0 International License, which permits use, sharing, adaptation, distribution and reproduction in any medium or format, as long as you give appropriate credit to the original author(s) and the source, provide a link to the Creative Commons license, and indicate if changes were made. The images or other third party material in this article are included in the article's Creative Commons license, unless indicated otherwise in a credit line to the material. If material is not included in the article's Creative Commons license and your intended use is not permitted by statutory regulation or exceeds the permitted use, you will need to obtain permission directly from the copyright holder. To view a copy of this license, visit http://creativecommons.org/licenses/by/4.0/.

(C) The Author(s) 2019 